\title{
CORONARY ARTERY DISEASE : AN EMERGING EPIDEMIC IN NEPAL
}

\author{
Maskey A*, Sayami A*, Pandey $M R^{* * *}$
}

\section{ABSTRACT}

Cardiovascular disease is the commonest cause of death globally and is the major contributor to the burden of premature mortality and morbidity. Coronary artery disease (CAD) is greatest killer of mankind. Coronary artery disease in western industrialized countries show a declining trend. However developing countries have shown an alarming increase. The identification of major risk factors and effective control of them through population based strategies of prevention were mainly responsible for this decline.A few cases of acute myocardial infarction were seen in Kathmandu ,Nepal in 1950 and in the sixties the incidence started rising rapidly. In a study between 1960-1968 a total of 150 cases were reported with myocardial infarction in Kathmandu , Nepal. Although national datas for incidence and prevalence on CAD in Nepal is not available, hospital based datas on admission pattern in different hospitals of Kathmandu, capital city of Nepal show 40 fold increase in incidence of acute myocardial infarction (AMI) in last 30 years. Prevalence of coronary artery disease (CAD) estimated from these figures of AMI cases indicate that $5 \%$ of adult population in Kathmandu suffer from CAD. Hence, Coronary artery disease is emerging as epidemic in Kathmandu, Nepal.

Key Words: Coronary artery disease (CAD), acute myocardial infarction (AMI), Epidemic.

\section{INTRODUCTION}

Cardiovascular diseases (CVD) accounts for approximately 12 millions deaths annually and is commonest cause of death globally. CVD is also the major contributor to the burden of premature mortality and morbidity and accounted for 85 millions disability adjusted (DALYs) life years in $1990 .{ }^{1}$ It has been estimated that during the past 25 years period from 1965-1990 coronary artery diseases (CAD) mortality fell by $50 \%$ in Australia, Canada, France \&US and 60\% in Japan and Finland. ${ }^{2}$ The identification of major risk factors and effective control of them through population based strategies of prevention were mainly responsible for this decline. CAD is the greatest killer of mankind. The rise and subsequent decline in CAD epidemic in almost all industrialized country in the later half of twentieth century has been well documented. However, developing countries have experienced alarming increase.

\section{GLOBAL SCENARIO}

Coronary artery disease(CAD) show a declining trend in Western industrialized countries. CAD was a rare cause of death in the US at the turn of the century, accounting for $<10 \%$ of all deaths in 1910. By 1965, me CAD mortalities rates increased more than five fold to $55 \%$ of all death, with only

* Shahid Gangalal National Heart Centre, Bansbari, Kathmandu, Nepal.

** Tribhuvan University Teaching Hospital, Kathmandu, Nepal.

*** Mrigendra Samjhana Medical Trust, Kathmandu, Nepal.

Address for correspondence : Dr. Arun Maskey

Shahid Gangalal National Heart Centre

Bansbari, Kathmandu, Nepal.

Email: maskeyarun@yahoo.com 
Finland having higher rates ${ }^{3}$ followed by sharp decline during the 25 years period from $1965-1990 .^{2}$ Over the past three decades, the annual decline of CAD was $0.6 \%$ for prevalence, $1-2 \%$ for incidence and $2-4 \%$ for mortality. ${ }^{4} \mathrm{CVD}$ is most common cause of death in China and Taiwan and the proportion of CVD death (particular CAD) has almost tripled from 12\%in 1957 to $36 \%$ in 1990.5 Japan has had the lowest rate of CAD among all industrialized countries for the past 50 years. Despite its affluences \& rapid urbanization, Japan has not only avoided the epidemic of CAD but also reduced their already low rate by $60 \% .{ }^{6}$ From 1960 to 1990 , the CAD prevalence increased from two fold (from $2 \%$ to $4 \%$ ) in rural India and three fold (from $3.45 \%$ to $9.45 \%$ ) in urban India. ${ }^{7}$

\section{SCENARIO IN NEPAL}

CAD is one of the common Cardiovascular diseases seen by physicians in their hospital \& private practice. Exact national data on incidence and prevalence of CAD in Nepal is not available. Smoking, Dyslipidaemia, Hypertension, Diabetes, physical inactivity are conventional risk factors. ${ }^{8}$ Different studies have shown high prevalence of these risk factors. The prevalence of hypertension in four different geographic regions of Nepal showed highest rate in urban communities ofKathmandu $(9.98 \%)$, followed by rural terai, Persaunni (8.11\%), rural hill Kathmandu, Bhadrabas (5.98\%), and the mountain region, Jumla (5.3\%). ${ }^{9}$ The incidence of hypertension for men and women in hilly rural village, Kotyand were 4.8 and $6.6 \%$ respectively and $14 \%$ and $8.1 \%$ respectively in suburban village Bhadrakali. ${ }^{10}$ Diabetes constituted $4 \%$ of total medical m-patients and their number is increasing. ${ }^{11}$ In a rural community of sundarijal and Bhanderbas panchayat of Kathmandu valley $68.7 \%$ were found to be smokers, $78.3 \%$ among male and $58.9 \%$ among female. This is of the highest prevalence rate of smoking habit reported from any where in me world. ${ }^{12}$ Definite diagnosis of myocardial infarction was made in Nepal in 1945. A few cases were seen in 1950 and in the sixties the incidence started rising rapidly. In a study between 1960-1968 a total of 150 cases were reported with myocardial infarction in Kathmandu, Nepal. Among them 89.9 were smokers, $28 \%$ hypertensive, $14.3 \%$ diabetics and $25.4 \%$ with hypercholesteraemia with male female ratio of $6.5 .{ }^{13} \mathrm{~A}$ study done 6 yrs back using Rose Questionnaire \& E.C.G. showed CAD among $4.8 \%$ of civil servants of Nepal above 35 yrs of age. ${ }^{14}$ In 1990, the admission pattern of Teaching hospital, Kathmandu showed respiratory diseases to be leading followed by gastrointestinal and cardiovascular diseases. However, in 2000 cardiovascular diseases constituted $20 \%$ of medical admission out of which $8 \%$ were CAD. The ten years data of teaching hospital among CAD patients showed $74 \%$ male and $26 \%$ female. Among them $82 \%$ were smokers, $40 \%$ hypertensive, $22 \%$ diabetic, $20 \%$ showed raised LDL and $10 \%$ showed raised triglyceride. In National heart centre Kathmandu CAD (21.7\%) was second most common cause of hospital admission following rheumatic heart disease (29.3\%)in first 300 cases. ${ }^{15}$ However, of 542 cases of total hospital admissions in national heart centre between 2001-2002, coronary artery disease was the commonest cause $(26.5 \%)$ followed by rheumatic heart disease (25.97\%). Currently an estimated acute myocardial infarction (AMI) cases admitted in 6 major hospitals of Katmandu totals 860 in a year. This amounts to 40 fold increase in incidence of AMI in last 30 years. Prevalence of coronary artery disease (CAD) estimated from the figures of AMI cases indicate that $5 \%$ of adult population in Kathmandu, capital city of Nepal suffer from CAD. As per definition of epidemic (an unusual occurrence in community of a disease clearly in excess of expected occurence), this disease reached epidemic proportion in at least Kathmandu valley, if not, of whole Nepal. ${ }^{16}$

In order to overcome the limitations of the currently available data, large international multicentre hospital based incident case control study of approximately 25000 cases of Acute myocardial infarction admitted in CCU are being conducted. Nepal is also actively participating in this Inter-Heart Study.

\section{CONCLUSION}

CAD is gradually emerging as one of the major health challenge in Nepal. The rapid change in life style, unhealthy habits (smoking, sedentary life style etc), economic development are considered to be responsible for the increase. Despite decrease in cardiovascular disease mortality in developed countries, substantial increase have been experienced in developing countries. Hence large scale epidemiological study should be carried out to determine the incidence \& prevalence of CAD in Nepal to identify the magnitude of the problem and timely primary and secondary prevention strategies should be vigorously pursued.

\section{REFERENCE}

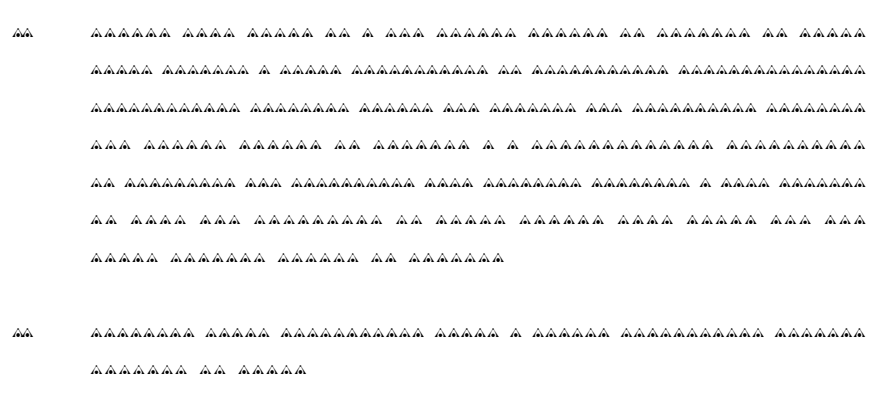




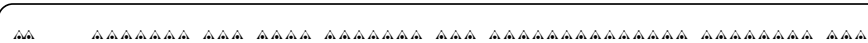

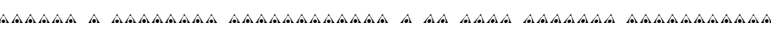

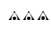

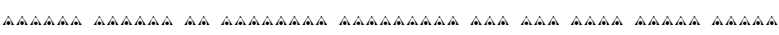

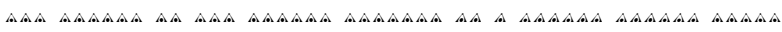

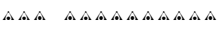

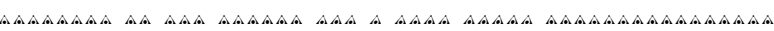
等

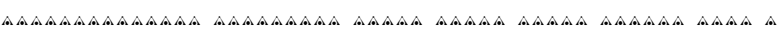
$\triangle A \triangle A \triangle A \triangle A \triangle A \triangle A \triangle A \triangle A$

ХАA

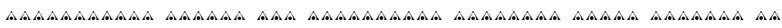

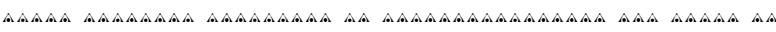

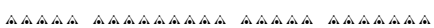

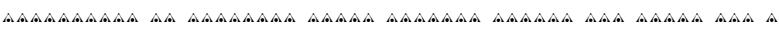

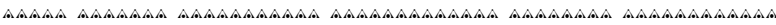

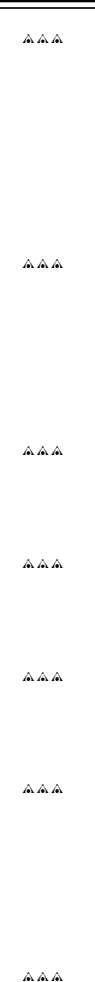

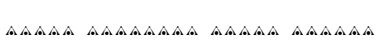

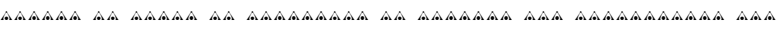

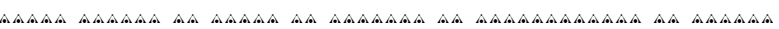

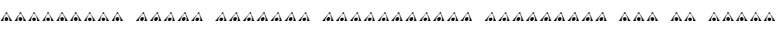
$\triangle A \triangle A \triangle A \triangle A \triangle A A$

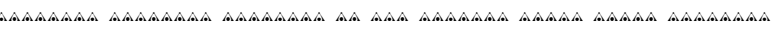

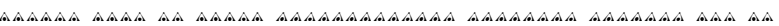
$\therefore A \Delta A \triangle A S A B$

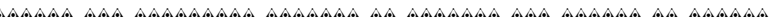

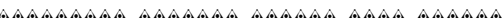

DA $\triangle A \triangle A A$ AAAAA AAAAAA ААA

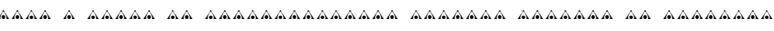

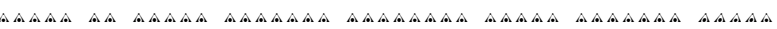
$\triangle A \triangle A \triangle A \triangle A \triangle A \triangle A \triangle A \triangle A$

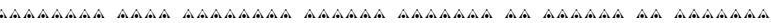

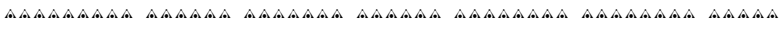

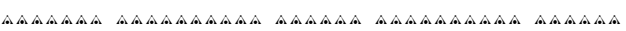

